\title{
Uncertainty Principles and Extremal Functions for the Dunkl $L^{2}$-Multiplier Operators
}

\author{
Fethi Soltani \\ Department of Mathematics, Faculty of Science, Jazan University, P.O. Box 114, Jazan, Saudi Arabia \\ Correspondence should be addressed to Fethi Soltani; fethisoltani10@yahoo.com
}

Received 19 November 2013; Accepted 12 June 2014; Published 17 August 2014

Academic Editor: Aref Jeribi

Copyright (c) 2014 Fethi Soltani. This is an open access article distributed under the Creative Commons Attribution License, which permits unrestricted use, distribution, and reproduction in any medium, provided the original work is properly cited.

We study some class of Dunkl $L^{2}$-multiplier operators; and related to these operators we establish the Heisenberg-Pauli-Weyl uncertainty principle and Donoho-Stark's uncertainty principle. We give also an application of the theory of reproducing kernels to the Tikhonov regularization on the Sobolev-Dunkl spaces.

\section{Introduction}

In this paper, we consider $\mathbb{R}^{d}$ with the Euclidean inner product $\langle\cdot, \cdot\rangle$ and norm $|y|:=\sqrt{\langle y, y\rangle}$. For $\alpha \in \mathbb{R}^{d} \backslash\{0\}$, let $\sigma_{\alpha}$ be the reflection in the hyperplane $H_{\alpha} \subset \mathbb{R}^{d}$ orthogonal to $\alpha$ :

$$
\sigma_{\alpha} x:=x-\frac{2\langle\alpha, x\rangle}{|\alpha|^{2}} \alpha
$$

A finite set $\Re \subset \mathbb{R}^{d} \backslash\{0\}$ is called a root system, if $\Re \cap$ R. $\alpha=\{-\alpha, \alpha\}$ and $\sigma_{\alpha} \mathfrak{R}=\Re$ for all $\alpha \in \mathfrak{R}$. We assume that it is normalized by $|\alpha|^{2}=2$ for all $\alpha \in \Re$. For a root system $\mathfrak{R}$, the reflections $\sigma_{\alpha}, \alpha \in \Re$, generate a finite group $G \subset$ $O(d)$, the reflection group associated with $\Re$. All reflections in $G$ correspond to suitable pairs of roots. For a given $\beta \in$ $\mathbb{R}^{d} \backslash \bigcup_{\alpha \in \mathfrak{R}} H_{\alpha}$, we fix the positive subsystem $\mathfrak{R}_{+}:=\{\alpha \in \mathfrak{R}$ : $\langle\alpha, \beta\rangle>0\}$. Then for each $\alpha \in \Re$ either $\alpha \in \mathfrak{R}_{+}$or $-\alpha \in \mathfrak{R}_{+}$.

Let $k: \Re \rightarrow \mathbb{C}$ be a multiplicity function on $\Re$ (i.e., a function which is constant on the orbits under the action of $G$ ). As an abbreviation, we introduce the index $\gamma=\gamma_{k}:=$ $\sum_{\alpha \in \Re_{+}} k(\alpha)$.

Throughout this paper, we will assume that $k(\alpha) \geq 0$ for all $\alpha \in \Re$. Moreover, let $w_{k}$ denote the weight function $w_{k}(x):=\prod_{\alpha \in \Re_{+}}|\langle\alpha, x\rangle|^{2 k(\alpha)}$, for all $x \in \mathbb{R}^{d}$, which is $G$ invariant and homogeneous of degree $2 \gamma$.
Let $c_{k}$ be the Mehta-type constant given by

$$
c_{k}:=\left(\int_{\mathbb{R}^{d}} e^{-|x|^{2} / 2} w_{k}(x) d x\right)^{-1} .
$$

We denote by $\mu_{k}$ the measure on $\mathbb{R}^{d}$ given by $d \mu_{k}(x):=$ $c_{k} w_{k}(x) d x$ and by $L^{p}\left(\mu_{k}\right), 1 \leq p \leq \infty$, the space of measurable functions $f$ on $\mathbb{R}^{d}$, such that

$$
\begin{gathered}
\|f\|_{L^{p}\left(\mu_{k}\right)}:=\left(\int_{\mathbb{R}^{d}}|f(x)|^{p} d \mu_{k}(x)\right)^{1 / p}<\infty, \\
1 \leq p<\infty, \\
\|f\|_{L^{\infty}\left(\mu_{k}\right)}:=\text { ess } \sup _{x \in \mathbb{R}^{d}}|f(x)|<\infty .
\end{gathered}
$$
by

For $f \in L^{1}\left(\mu_{k}\right)$ the Dunkl transform is defined (see [1])

$$
\begin{array}{r}
\mathscr{F}_{k}(f)(y):=\int_{\mathbb{R}^{d}} E_{k}(-i x, y) f(x) d \mu_{k}(x), \\
y \in \mathbb{R}^{d},
\end{array}
$$

where $E_{k}(-i x, y)$ denotes the Dunkl kernel (for more details, see Section 2).

Many uncertainty principles have already been proved for the Dunkl transform, namely, by Rösler [2] and Shimeno [3] 
who established the Heisenberg-Pauli-Weyl inequality for the Dunkl transform, by showing that, for every $f \in L^{2}\left(\mu_{k}\right)$,

$$
\|f\|_{L^{2}\left(\mu_{k}\right)}^{2} \leq \frac{2}{2 \gamma+d}\||x| f\|_{L^{2}\left(\mu_{k}\right)}\left\||y| \mathscr{F}_{k}(f)\right\|_{L^{2}\left(\mu_{k}\right)} .
$$

Recently, the author $[4,5]$ proved general forms of the Heisenberg-Pauli-Weyl inequality for the Dunkl transform.

Let $m$ be a function in $L^{2}\left(\mu_{k}\right)$. The Dunkl $L^{2}$-multiplier operators, $T_{k, m}$, are defined, for regular functions $f$ on $\mathbb{R}^{d}$, by

$$
\begin{array}{r}
T_{k, m} f(a, x):=\mathscr{F}_{k}^{-1}\left(m(a x) \mathscr{F}_{k}(f)\right)(x), \\
(a, x) \in] 0, \infty\left[\times \mathbb{R}^{d} .\right.
\end{array}
$$

These operators are studied in $[6,7]$ where the author established some applications (Calderón's reproducing formulas, best approximation formulas, and extremal functions...).

For $m \in L^{2}\left(\mu_{k}\right)$ satisfying the admissibility condition: $\int_{0}^{\infty}|m(a x)|^{2}(d a / a)=1$, a.e. $x \in \mathbb{R}^{d}$, then the operators $T_{k, m}$ satisfy Plancherel's formula:

$$
\begin{array}{r}
\left\|T_{k, m} f\right\|_{L^{2}\left(\Omega_{k}\right)}^{2}:=\int_{\mathbb{R}^{d}} \int_{0}^{\infty}\left|T_{k, m} f(a, x)\right|^{2} d \Omega_{k}(a, x)=\|f\|_{L^{2}\left(\mu_{k}\right)}^{2}, \\
f \in L^{2}\left(\mu_{k}\right),
\end{array}
$$

where $\Omega_{k}$ is the measure on $] 0, \infty\left[\times \mathbb{R}^{d}\right.$ given by $d \Omega_{k}(a, x):=$ $(d a / a) d \mu_{k}(x)$.

For the operators $T_{k, m}$ we establish a Heisenberg-PauliWeyl uncertainty principle. More precisely, we will show, for $f \in L^{2}\left(\mu_{k}\right)$,

$$
\begin{aligned}
& \|f\|_{L^{2}\left(\mu_{k}\right)}^{2} \\
& \leq \frac{2}{2 \gamma+d}\left\||y| \mathscr{F}_{k}(f)\right\|_{L^{2}\left(\mu_{k}\right)} \\
& \quad \cdot\left(\int_{\mathbb{R}^{d}} \int_{0}^{\infty}|x|^{2}\left|T_{k, m} f(a, x)\right|^{2} \frac{d a}{a} d \mu_{k}(x)\right)^{1 / 2},
\end{aligned}
$$

provided $m \in L^{2}\left(\mu_{k}\right)$ satisfying $\int_{0}^{\infty}|m(a x)|^{2}(d a / a)=1$, a.e. $x \in \mathbb{R}^{d}$.

Building on the techniques of Donoho and Stark [8], we show a continuous-time principle for the $L^{2}$ theory. Let $E$ be measurable subset of $\mathbb{R}^{d}$, let $S$ be measurable subset of ] $0, \infty\left[\times \mathbb{R}^{d}\right.$, and let $f \in L^{2}\left(\mu_{k}\right)$. If $f$ is $\varepsilon$-concentrated on $E$ and $T_{k, m} f$ is $\eta$-concentrated on $S$ (see Section 3 for more details), then

$$
\left(\mu_{k}(E)\right)^{1 / 2}\left(\iint_{S} \frac{1}{a^{2(2 \gamma+d)}} d \Omega_{k}(a, x)\right)^{1 / 2} \geq \frac{1-\eta-\varepsilon}{\|m\|_{L^{1}\left(\mu_{k}\right)}},
$$

provided $m \in L^{1} \cap L^{2}\left(\mu_{k}\right)$ satisfying $\int_{0}^{\infty}|m(a x)|^{2}(d a / a)=1$, a.e. $x \in \mathbb{R}^{d}$.

Building on the ideas of Saitoh [9, 10], Matsuura et al. [11], and Yamada et al. [12], we give an application of the theory of reproducing kernels to the Tikhonov regularization, which gives the best approximation of the operator $T_{k, m}$ on the Sobolev-Dunkl spaces $H^{s}\left(\mu_{k}\right)$. More precisely, for all $\lambda>$ $0, g \in L^{2}\left(\Omega_{k}\right)$, the infimum

$$
\inf _{f \in H^{s}\left(\mu_{k}\right)}\left\{\lambda\|f\|_{H^{s}\left(\mu_{k}\right)}^{2}+\left\|g-T_{k, m} f\right\|_{L^{2}\left(\Omega_{k}\right)}^{2}\right\}
$$

is attained at one function $f_{\lambda, g}^{*}$, called the extremal function.

In particular for $f \in H^{s}\left(\mu_{k}\right)$ and $g=T_{k, m} f$, the corresponding extremal functions $\left\{f_{\lambda}^{*}\right\}_{\lambda>0}$ converge to $f$ as $\lambda \rightarrow 0^{+}$.

This paper is organized as follows. In Section 2 we define the Dunkl $L^{2}$-multiplier operators $T_{k, m}$, and we give for them Plancherel's formula. Some examples of Dunkl $L^{2}$-multiplier operators are given. In Section 3 we establish the HeisenbergPauli-Weyl uncertainty principle and Donoho-Stark's uncertainty principle for the operators $T_{k, m}$. In the last section we give an application of the theory of reproducing kernels to the Tikhonov regularization related to the operators $T_{k, m}$ on the Sobolev-Dunkl spaces $H^{s}\left(\mu_{k}\right)$.

\section{The Dunkl $L^{2}$-Multiplier Operators on $\mathbb{R}^{d}$}

The Dunkl operators $\mathscr{D}_{j}, j=1, \ldots, d$, on $\mathbb{R}^{d}$ associated with the finite reflection group $G$ and multiplicity function $k$ are given, for a function $f$ of class $C^{1}$ on $\mathbb{R}^{d}$, by

$$
\mathscr{D}_{j} f(x):=\frac{\partial}{\partial x_{j}} f(x)+\sum_{\alpha \in \Re_{+}} k(\alpha) \alpha_{j} \frac{f(x)-f\left(\sigma_{\alpha} x\right)}{\langle\alpha, x\rangle} .
$$

For $y \in \mathbb{R}^{d}$, the initial problem $\mathscr{D}_{j} u(\cdot, y)(x)=y_{j} u(x, y)$, $j=1, \ldots, d$, with $u(0, y)=1$ admits a unique analytic solution on $\mathbb{R}^{d}$, which will be denoted by $E_{k}(x, y)$ and called Dunkl kernel $[13,14]$. This kernel has a unique analytic extension to $\mathbb{C}^{d} \times \mathbb{C}^{d}$ (see [15]). In our case (see $[1,13]$ ),

$$
\left|E_{k}(i x, y)\right| \leq 1, \quad x, y \in \mathbb{R}^{d} .
$$

The Dunkl kernel gives rise to an integral transform, which is called Dunkl transform on $\mathbb{R}^{d}$, and was introduced by Dunkl in [1], where already many basic properties were established. Dunkl's results were completed and extended later by de Jeu [14]. The Dunkl transform of a function $f$, in $L^{1}\left(\mu_{k}\right)$, is defined by

$$
\begin{array}{r}
\mathscr{F}_{k}(f)(y):=\int_{\mathbb{R}^{d}} E_{k}(-i x, y) f(x) d \mu_{k}(x), \\
y \in \mathbb{R}^{d} .
\end{array}
$$

We notice that $\mathscr{F}_{0}$ agrees with the Fourier transform $\mathscr{F}$ that is given by

$$
\mathscr{F}(f)(y):=(2 \pi)^{-d / 2} \int_{\mathbb{R}^{d}} e^{-i\langle x, y\rangle} f(x) d x, \quad x \in \mathbb{R}^{d} .
$$

Some of the properties of Dunkl transform $\mathscr{F}_{k}$ are collected bellow (see $[1,14]$ ). 
Theorem 1. (i) $L^{1}-L^{\infty}$-boundedness: for all $f \in L^{1}\left(\mu_{k}\right)$, $\mathscr{F}_{k}(f) \in L^{\infty}\left(\mu_{k}\right)$ and

$$
\left\|\mathscr{F}_{k}(f)\right\|_{L^{\infty}\left(\mu_{k}\right)} \leq\|f\|_{L^{1}\left(\mu_{k}\right)} .
$$

(ii) Inversion theorem: let $f \in L^{1}\left(\mu_{k}\right)$, such that $\mathscr{F}_{k}(f) \in$ $L^{1}\left(\mu_{k}\right)$. Then

$$
f(x)=\mathscr{F}_{k}\left(\mathscr{F}_{k}(f)\right)(-x) \text {, a.e. } x \in \mathbb{R}^{d} .
$$

(iii) Plancherel theorem: the Dunkl transform $\mathscr{F}_{k}$ extends uniquely to an isometric isomorphism of $L^{2}\left(\mu_{k}\right)$ onto itself. In particular,

$$
\left\|\mathscr{F}_{k}(f)\right\|_{L^{2}\left(\mu_{k}\right)}=\|f\|_{L^{2}\left(\mu_{k}\right)} .
$$

Let $m$ be a function in $L^{2}\left(\mu_{k}\right)$. The Dunkl $L^{2}$-multiplier operators, $T_{k, m}$, are defined, for regular functions $f$ on $\mathbb{R}^{d}$, by

$$
\begin{array}{r}
T_{k, m} f(a, x):=\mathscr{F}_{k}^{-1}\left(m(a x) \mathscr{F}_{k}(f)\right)(x), \\
(a, x) \in] 0, \infty\left[\times \mathbb{R}^{d} .\right.
\end{array}
$$

The operators $T_{k, m}$ satisfy the following integral representation.

Lemma 2. If $m, f \in L^{1} \cap L^{2}\left(\mu_{k}\right)$, then

$$
\begin{array}{r}
T_{k, m} f(a, x)=\frac{1}{a^{2 \gamma+d}} \int_{\mathbb{R}^{d}} H_{k}\left(\frac{x}{a}, \frac{y}{a}, m\right) f(y) d \mu_{k}(y), \\
(a, x) \in] 0, \infty\left[\times \mathbb{R}^{d},\right.
\end{array}
$$

where

$$
\begin{aligned}
& H_{k}(x, y, m) \\
& \quad=\int_{\mathbb{R}^{d}} m(z) E_{k}(i x, z) E_{k}(-i y, z) d \mu_{k}(z) .
\end{aligned}
$$

Proof. The result follows from (18) and Theorem 1(ii) using Fubini-Tonnelli's theorem.

We denote by $\Omega_{k}$ the measure on $] 0, \infty\left[\times \mathbb{R}^{d}\right.$ given by $d \Omega_{k}(a, x):=(d a / a) d \mu_{k}(x)$ and by $L^{2}\left(\Omega_{k}\right)$ the space of measurable functions $F$ on $] 0, \infty\left[\times \mathbb{R}^{d}\right.$, such that

$$
\|F\|_{L^{2}\left(\Omega_{k}\right)}:=\left(\int_{\mathbb{R}^{d}} \int_{0}^{\infty}|F(a, x)|^{2} d \Omega_{k}(a, x)\right)^{1 / 2}<\infty .
$$

In the following, we give Plancherel formula for the operators $T_{k, m}$.

Theorem 3 (Plancherel formula). Let $m$ be a function in $L^{2}\left(\mu_{k}\right)$ satisfying the admissibility condition:

$$
\int_{0}^{\infty}|m(a x)|^{2} \frac{d a}{a}=1, \quad \text { a.e. } x \in \mathbb{R}^{d} .
$$

Then, for $f \in L^{2}\left(\mu_{k}\right)$, one has

$$
\left\|T_{k, m} f\right\|_{L^{2}\left(\Omega_{k}\right)}=\|f\|_{L^{2}\left(\mu_{k}\right)} .
$$

Proof. From Fubini-Tonnelli's theorem, we obtain

$$
\begin{aligned}
& \int_{\mathbb{R}^{d}} \int_{0}^{\infty}\left|T_{k, m} f(a, x)\right|^{2} d \Omega_{k}(a, x) \\
& =\int_{0}^{\infty} \int_{\mathbb{R}^{d}}|m(a y)|^{2}\left|\mathscr{F}_{k}(f)(y)\right|^{2} d \mu_{k}(y) \frac{d a}{a} \\
& =\int_{\mathbb{R}^{d}}\left|\mathscr{F}_{k}(f)(y)\right|^{2}\left[\int_{0}^{\infty}|m(a y)|^{2} \frac{d a}{a}\right] d \mu_{k}(y) .
\end{aligned}
$$

Then, the result follows from (22) and Theorem 1(iii).

As applications, we give the following examples.

Example 4. Let $m_{t}, t>0$, the function is defined by

$$
m_{t}(x):=-\sqrt{8} t|x|^{2} e^{-t|x|^{2}}, \quad x \in \mathbb{R}^{d} .
$$

Then

(a) $m_{t}$ belongs to $L^{1} \cap L^{2}\left(\mu_{k}\right)$, and by (2), we have

$$
\begin{aligned}
\left\|m_{t}\right\|_{L^{1}\left(\mu_{k}\right)} & =\sqrt{8} t \int_{\mathbb{R}^{d}}|x|^{2} e^{-t|x|^{2}} d \mu_{k}(x) \\
& =-\sqrt{8} t \frac{\partial}{\partial t}\left(\int_{\mathbb{R}^{d}} e^{-t|x|^{2}} d \mu_{k}(x)\right) \\
& =\frac{\sqrt{2}(2 \gamma+d)}{(\sqrt{2 t})^{2 \gamma+d}} \\
\left\|m_{t}\right\|_{L^{2}\left(\mu_{k}\right)}^{2} & =8 t^{2} \int_{\mathbb{R}^{d}}|x|^{4} e^{-2 t|x|^{2}} d \mu_{k}(x) \\
& =2 t^{2} \frac{\partial^{2}}{\partial t^{2}}\left(\int_{\mathbb{R}^{d}} e^{-2 t|x|^{2}} d \mu_{k}(x)\right) \\
& =\frac{(2 \gamma+d)(\gamma+d / 2+1)}{(2 \sqrt{t})^{2 \gamma+d}} .
\end{aligned}
$$

(b) $m_{t}$ satisfies the admissibility condition (22), that is,

$$
\int_{0}^{\infty}\left|m_{t}(a x)\right|^{2} \frac{d a}{a}=8 t^{2}|x|^{4} \int_{0}^{\infty} a^{3} e^{-2 t|x|^{2} a^{2}} d a=1 .
$$

Then the associated operators $T_{k, m_{t}}$ satisfy Plancherel's formula (23).

To express the operator $T_{k, m_{t}}$, we use Lemma 2 , then for $f \in L^{1} \cap L^{2}\left(\mu_{k}\right)$, we have

$$
\begin{array}{r}
T_{k, m_{t}} f(a, x)=\frac{\sqrt{8} t}{a^{2}+d} \int_{\mathbb{R}^{d}} \frac{\partial}{\partial t}\left[h_{k}\left(\frac{x}{a}, \frac{y}{a}, t\right)\right] f(y) d \mu_{k}(y), \\
x \in \mathbb{R}^{d},
\end{array}
$$

where

$$
h_{k}(x, y, t)=\int_{\mathbb{R}^{d}} e^{-t|z|^{2}} E_{k}(i x, z) E_{k}(-i y, z) d \mu_{k}(z)
$$


is the Dunkl-type heat kernel $[16,17]$. From [16] this kernel is given by

$$
h_{k}(x, y, t)=\frac{1}{(2 t)^{\gamma+d / 2}} e^{-\left(|x|^{2}+|y|^{2}\right) / 4 t} E_{k}\left(\frac{x}{\sqrt{2 t}}, \frac{y}{\sqrt{2 t}}\right) .
$$

Example 5. Let $m_{t}, t>0$, be the function defined by

$$
m_{t}(x):=-2 t|x| e^{-t|x|}, \quad x \in \mathbb{R}^{d} .
$$

Then one has:

(a) $m_{t}$ belongs to $L^{1} \cap L^{2}\left(\mu_{k}\right)$, and

$$
\begin{aligned}
\left\|m_{t}\right\|_{L^{1}\left(\mu_{k}\right)} & =2 t \int_{\mathbb{R}^{d}}|x| e^{-t|x|} d \mu_{k}(x) \\
& =-2 t \frac{\partial}{\partial t}\left(\int_{\mathbb{R}^{d}} e^{-t|x|} d \mu_{k}(x)\right) .
\end{aligned}
$$

Since

$$
e^{-t|x|}=\frac{1}{\sqrt{\pi}} \int_{0}^{\infty} \frac{e^{-s}}{\sqrt{s}} e^{-\left(t^{2} / 4 s\right)|x|^{2}} d s,
$$

by Fubini-Tonnelli's theorem and (2), we deduce that

$$
\begin{aligned}
\int_{\mathbb{R}^{d}} e^{-t|x|} d \mu_{k}(x) \\
=\frac{1}{\sqrt{\pi}} \int_{0}^{\infty} \frac{e^{-s}}{\sqrt{s}}\left(\int_{\mathbb{R}^{d}} e^{-\left(t^{2} / 4 s\right)|x|^{2}} d \mu_{k}(x)\right) d s \\
=\frac{1}{\sqrt{\pi}} \int_{0}^{\infty} \frac{e^{-s}}{\sqrt{s}}\left(\frac{\sqrt{2 s}}{t}\right)^{2 \gamma+d} d s \\
=\frac{\Gamma(\gamma+(d+1) / 2)}{\sqrt{\pi}}\left(\frac{\sqrt{2}}{t}\right)^{2 \gamma+d} .
\end{aligned}
$$

Thus,

$$
\left\|m_{t}\right\|_{L^{1}\left(\mu_{k}\right)}=\frac{2(2 \gamma+d) \Gamma(\gamma+(d+1) / 2)}{\sqrt{\pi}}\left(\frac{\sqrt{2}}{t}\right)^{2 \gamma+d} .
$$

On the other hand,

$$
\begin{aligned}
\left\|m_{t}\right\|_{L^{2}\left(\mu_{k}\right)}^{2} & =4 t^{2} \int_{\mathbb{R}^{d}}|x|^{2} e^{-2 t|x|} d \mu_{k}(x) \\
& =\frac{\partial^{2}}{\partial t^{2}}\left(\int_{\mathbb{R}^{d}} e^{-2 t|x|} d \mu_{k}(x)\right) \\
& =\frac{\partial^{2}}{\partial t^{2}}\left(\frac{\Gamma(\gamma+(d+1) / 2)}{\sqrt{\pi}(\sqrt{2} t)^{2 \gamma+d}}\right) .
\end{aligned}
$$

Thus,

$$
\left\|m_{t}\right\|_{L^{2}\left(\mu_{k}\right)}^{2}=\frac{4(2 \gamma+d) \Gamma(\gamma+(d+3) / 2)}{\sqrt{\pi}(\sqrt{2} t)^{2 \gamma+d+2}} .
$$

(b) $m_{t}$ satisfies the admissibility condition (22); that is,

$$
\int_{0}^{\infty}\left|m_{t}(a x)\right|^{2} \frac{d a}{a}=4 t^{2}|x|^{2} \int_{0}^{\infty} a e^{-2 t|x| a} d a=1
$$

Then the associated operators $T_{k, m_{t}}$ satisfy Plancherel's formula (23).

To express the operators $T_{k, m_{t}}$, we use Lemma 2; then for $f \in L^{1} \cap L^{2}\left(\mu_{k}\right)$, we have

$$
\begin{aligned}
& T_{k, m_{t}} f(a, x) \\
& \quad=\frac{2 t}{a^{2} \gamma+d} \int_{\mathbb{R}^{d}} \frac{\partial}{\partial t}\left[p_{k}\left(\frac{x}{a}, \frac{y}{a}, t\right)\right] f(y) d \mu_{k}(y),
\end{aligned}
$$

where

$$
p_{k}(x, y, t)=\int_{\mathbb{R}^{d}} e^{-t|z|} E_{k}(i x, z) E_{k}(-i y, z) d \mu_{k}(z)
$$

is the Dunkl-type Poisson kernel [18]. From (33) this kernel is given by

$$
p_{k}(x, y, t)=\frac{1}{\sqrt{\pi}} \int_{0}^{\infty} \frac{e^{-s}}{\sqrt{s}} h_{k}\left(x, y, \frac{t^{2}}{4 s}\right) d s .
$$

\section{Uncertainty Principle for the Operators $T_{k, m}$}

3.1. Heisenberg-Pauli-Weyl Uncertainty Principle. This section is devoted to establish Heisenberg-Pauli-Weyl uncertainty principle for the operators $T_{k, m}$; more precisely, we will show the following theorem.

Theorem 6. Let $m$ be a function in $L^{2}\left(\mu_{k}\right)$ satisfying the admissibility condition (22). Then, for $f \in L^{2}\left(\mu_{k}\right)$, one has

$$
\begin{aligned}
\|f\|_{L^{2}\left(\mu_{k}\right)}^{2} \leq & \frac{2}{2 \gamma+d}\left\|y \mid \mathscr{F}_{k}(f)\right\|_{L^{2}\left(\mu_{k}\right)} \\
& \cdot\left(\int_{\mathbb{R}^{d}} \int_{0}^{\infty}|x|^{2}\left|T_{k, m} f(a, x)\right|^{2} \frac{d a}{a} d \mu_{k}(x)\right)^{1 / 2} .
\end{aligned}
$$

Proof. Let $f \in L^{2}\left(\mu_{k}\right)$

Assume that $\left\||y| \mathscr{F}_{k}(f)\right\|_{L^{2}\left(\mu_{k}\right)}<\infty$ and $\int_{\mathbb{R}^{d}} \int_{0}^{\infty}|x|^{2}\left|T_{k, m} f(a, x)\right|^{2}(d a / a) d \mu_{k}(x)<\infty$. The inequality (5) leads to

$$
\begin{aligned}
\int_{\mathbb{R}^{d}}\left|T_{k, m} f(a, x)\right|^{2} d \mu_{k}(x) \\
\leq \frac{2}{2 \gamma+d}\left(\int_{\mathbb{R}^{d}}|x|^{2}\left|T_{k, m} f(a, x)\right|^{2} d \mu_{k}(x)\right)^{1 / 2} \\
\quad \cdot\left(\int_{\mathbb{R}^{d}}|y|^{2}\left|\mathscr{F}_{k}\left(T_{k, m} f(a, \cdot)\right)(y)\right|^{2} d \mu_{k}(y)\right)^{1 / 2} \cdot
\end{aligned}
$$


Integrating with respect to $(d a / a)$ gives

$$
\begin{aligned}
& \left\|T_{k, m} f\right\|_{L^{2}\left(\Omega_{k}\right)}^{2} \\
& \leq \frac{2}{2 \gamma+d} \int_{0}^{\infty}\left(\int_{\mathbb{R}^{d}}|x|^{2}\left|T_{k, m} f(a, x)\right|^{2} d \mu_{k}(x)\right)^{1 / 2} \\
& \quad \cdot\left(\int_{\mathbb{R}^{d}}|y|^{2}\left|\mathscr{F}_{k}\left(T_{k, m} f(a, \cdot)\right)(y)\right|^{2} d \mu_{k}(y)\right)^{1 / 2} \frac{d a}{a} .
\end{aligned}
$$

From Theorem 3 and Schwarz's inequality, we get

$$
\begin{aligned}
\|f\|_{L^{2}\left(\mu_{k}\right)}^{2} & \leq \frac{2}{2 \gamma+d}\left(\int_{0}^{\infty} \int_{\mathbb{R}^{d}}|x|^{2}\left|T_{k, m} f(a, x)\right|^{2} d \mu_{k}(x) \frac{d a}{a}\right)^{1 / 2} \\
& \cdot\left(\int_{0}^{\infty} \int_{\mathbb{R}^{d}}|y|^{2}\left|\mathscr{F}_{k}\left(T_{k, m} f(a, \cdot)\right)(y)\right|^{2} d \mu_{k}(y) \frac{d a}{a}\right)^{1 / 2} .
\end{aligned}
$$

But by (18), Fubini-Tonnelli's theorem, and (22), we have

$$
\begin{aligned}
\int_{0}^{\infty} & \int_{\mathbb{R}^{d}}|y|^{2}\left|\mathscr{F}_{k}\left(T_{k, m} f(a, \cdot)\right)(y)\right|^{2} d \mu_{k}(y) \frac{d a}{a} \\
= & \int_{0}^{\infty} \int_{\mathbb{R}^{d}}|y|^{2}|m(a y)|^{2}\left|\mathscr{F}_{k}(f)(y)\right|^{2} d \mu_{k}(y) \frac{d a}{a} \\
= & \int_{\mathbb{R}^{d}}|y|^{2}\left|\mathscr{F}_{k}(f)(y)\right|^{2} d \mu_{k}(y) .
\end{aligned}
$$

This yields the result and completes the proof of the theorem.

3.2. Uncertainty Principle of Concentration Type. Let $E$ be a measurable subset of $\mathbb{R}^{d}$. We say that a function $f \in L^{2}\left(\mu_{k}\right)$ is $\mathcal{E}$-concentrated on $E$, if

$$
\left\|f-\chi_{E} f\right\|_{L^{2}\left(\mu_{k}\right)} \leq \varepsilon\|f\|_{L^{2}\left(\mu_{k}\right)}
$$

where $\chi_{E}$ is the indicator function of the set $E$.

Let $S$ be a measurable subset of $] 0, \infty\left[\times \mathbb{R}^{d}\right.$ and let $f \in$ $L^{2}\left(\mu_{k}\right)$. We say that $T_{k, m} f$ is $\eta$-concentrated on $S$, if

$$
\left\|T_{k, m} f-\chi_{S} T_{k, m} f\right\|_{L^{2}\left(\Omega_{k}\right)} \leq \eta\left\|T_{k, m} f\right\|_{L^{2}\left(\Omega_{k}\right)} .
$$

Donoho-Stark's uncertainty principle for the operators $T_{k, m}$ is obtained.

Theorem 7. Let $f \in L^{2}\left(\mu_{k}\right)$ and let $m \in L^{1} \cap L^{2}\left(\mu_{k}\right)$ satisfying (22). If $f$ is $\varepsilon$-concentrated on $E$ and $T_{k, m} f$ is $\eta$-concentrated on $S$, then

$$
\begin{aligned}
& \|m\|_{L^{1}\left(\mu_{k}\right)}\left(\mu_{k}(E)\right)^{1 / 2}\left(\iint_{S} \frac{1}{a^{2(2 \gamma+d)}} d \Omega_{k}(a, x)\right)^{1 / 2} \\
& \quad \geq 1-\eta-\varepsilon .
\end{aligned}
$$

Proof. Let $f \in L^{2}\left(\mu_{k}\right)$. Assume that $\mu_{k}(E)<\infty$ and $\iint_{S}\left(1 / a^{2(2 \gamma+d)}\right) d \Omega_{k}(a, x)<\infty$. From (47), (48), and Theorem 3 it follows that

$$
\begin{aligned}
& \left\|T_{k, m} f-\chi_{S} T_{k, m}\left(\chi_{E} f\right)\right\|_{L^{2}\left(\Omega_{k}\right)} \\
& \leq\left\|T_{k, m} f-\chi_{S} T_{k, m} f\right\|_{L^{2}\left(\Omega_{k}\right)} \\
& \quad+\left\|\chi_{S} T_{k, m}\left(f-\chi_{E} f\right)\right\|_{L^{2}\left(\Omega_{k}\right)} \\
& \leq \eta\left\|T_{k, m} f\right\|_{L^{2}\left(\Omega_{k}\right)} \\
& \quad+\left\|T_{k, m}\left(f-\chi_{E} f\right)\right\|_{L^{2}\left(\Omega_{k}\right)} \\
& \leq(\eta+\varepsilon)\|f\|_{L^{2}\left(\mu_{k}\right)} .
\end{aligned}
$$

Then the triangle inequality shows that

$$
\begin{aligned}
\left\|T_{k, m} f\right\|_{L^{2}\left(\Omega_{k}\right)} \leq & \left\|\chi_{S} T_{k, m}\left(\chi_{E} f\right)\right\|_{L^{2}\left(\Omega_{k}\right)} \\
& +\left\|T_{k, m} f-\chi_{S} T_{k, m}\left(\chi_{E} f\right)\right\|_{L^{2}\left(\Omega_{k}\right)} \\
\leq & \left\|\chi_{S} T_{k, m}\left(\chi_{E} f\right)\right\|_{L^{2}\left(\Omega_{k}\right)} \\
& +(\eta+\varepsilon)\|f\|_{L^{2}\left(\mu_{k}\right)} .
\end{aligned}
$$

But

$$
\begin{aligned}
& \left\|\chi_{S} T_{k, m}\left(\chi_{E} f\right)\right\|_{L^{2}\left(\Omega_{k}\right)} \\
& \quad=\left(\iint_{S}\left|T_{k, m}\left(\chi_{E} f\right)(a, x)\right|^{2} d \Omega_{k}(a, x)\right)^{1 / 2}
\end{aligned}
$$

and since $m, \chi_{E} f \in L^{1} \cap L^{2}\left(\mu_{k}\right)$, then, by Lemma 2 , we have

$$
\begin{aligned}
& \left|T_{k, m}\left(\chi_{E} f\right)(a, x)\right| \\
& \quad \leq \frac{1}{a^{2 \gamma+d}}\|m\|_{L^{1}\left(\mu_{k}\right)}\|f\|_{L^{2}\left(\mu_{k}\right)}\left(\mu_{k}(E)\right)^{1 / 2} .
\end{aligned}
$$

Thus,

$$
\begin{gathered}
\left\|\chi_{S} T_{k, m}\left(\chi_{E} f\right)\right\|_{L^{2}\left(\Omega_{k}\right)} \\
\leq\|m\|_{L^{1}\left(\mu_{k}\right)}\|f\|_{L^{2}\left(\mu_{k}\right)}\left(\mu_{k}(E)\right)^{1 / 2} \\
\cdot\left(\iint_{S} \frac{1}{a^{2(2 \gamma+d)}} d \Omega_{k}(a, x)\right)^{1 / 2}, \\
\left\|T_{k, m} f\right\|_{L^{2}\left(\Omega_{k}\right)} \\
\leq\|m\|_{L^{1}\left(\mu_{k}\right)}\|f\|_{L^{2}\left(\mu_{k}\right)}\left(\mu_{k}(E)\right)^{1 / 2} \\
\cdot\left(\iint_{S} \frac{1}{a^{2(2 \gamma+d)}} d \Omega_{k}(a, x)\right)^{1 / 2} \\
+(\eta+\varepsilon)\|f\|_{L^{2}\left(\mu_{k}\right)} .
\end{gathered}
$$

By applying Theorem 3, we obtain

$$
\begin{aligned}
& \|m\|_{L^{1}\left(\mu_{k}\right)}\left(\mu_{k}(E)\right)^{1 / 2}\left(\iint_{S} \frac{1}{a^{2(2 \gamma+d)}} d \Omega_{k}(a, x)\right)^{1 / 2} \\
& \quad \geq 1-\eta-\varepsilon,
\end{aligned}
$$

which gives the desired result. 
Remark 8. If $S \subset\{(a, x) \in] 0, \infty\left[\times \mathbb{R}^{d}: a \geq \delta\right\}$ for some $\delta>0$, one supposes that $\alpha=\max \{1 / a:(a, x) \in S$ for some $x \in$ $\left.\mathbb{R}^{d}\right\}$. Then by Theorem 7 we deduce that

$$
\alpha^{2 \gamma+d}\|m\|_{L^{1}\left(\mu_{k}\right)}\left(\mu_{k}(E)\right)^{1 / 2}\left(\Omega_{k}(S)\right)^{1 / 2} \geq 1-\eta-\varepsilon .
$$

\section{Extremal Functions for the Operators $T_{k, m}$}

4.1. Sobolev-Dunkl Spaces. Let $s \geq 0$. We define the SobolevDunkl space of order $s$, which will be denoted by $H^{s}\left(\mu_{k}\right)$, as the set of all $f \in L^{2}\left(\mu_{k}\right)$ such that $\left(1+|z|^{2}\right)^{s / 2} \mathscr{F}_{k}(f) \in L^{2}\left(\mu_{k}\right)$. The space $H^{s}\left(\mu_{k}\right)$ is provided with the inner product

$$
\begin{aligned}
& \langle f, g\rangle_{H^{s}\left(\mu_{k}\right)} \\
& \quad=\int_{\mathbb{R}^{d}}\left(1+|z|^{2}\right)^{s} \mathscr{F}_{k}(f)(z) \overline{\mathscr{F}_{k}(g)(z)} d \mu_{k}(z)
\end{aligned}
$$

and the norm

$$
\|f\|_{H^{s}\left(\mu_{k}\right)}=\left[\int_{\mathbb{R}^{d}}\left(1+|z|^{2}\right)^{s}\left|\mathscr{F}_{k}(f)(z)\right|^{2} d \mu_{k}(z)\right]^{1 / 2} .
$$

The space $H^{s}\left(\mu_{k}\right)$ satisfies the following properties.

(a) Consider $H^{0}\left(\mu_{k}\right)=L^{2}\left(\mu_{k}\right)$.

(b) For all $s>0$, the space $H^{s}\left(\mu_{k}\right)$ is continuously contained in $L^{2}\left(\mu_{k}\right)$ and $\|f\|_{L^{2}\left(\mu_{k}\right)} \leq\|f\|_{H^{s}\left(\mu_{k}\right)}$.

(c) For all $s, t>0$, such that $t>s$, the space $H^{t}\left(\mu_{k}\right)$ is continuously contained in $H^{s}\left(\mu_{k}\right)$ and $\|f\|_{H^{s}\left(\mu_{k}\right)} \leq$ $\|f\|_{H^{t}\left(\mu_{k}\right)}$.

(d) The space $H^{s}\left(\mu_{k}\right), s \geq 0$, provided with the inner product $\langle\cdot, \cdot\rangle_{H^{s}\left(\mu_{k}\right)}$ is a Hilbert space.

Remark 9. For $s>\gamma+d / 2$, the function $y \rightarrow\left(1+|z|^{2}\right)^{-s / 2}$ belongs to $L^{2}\left(\mu_{k}\right)$.

Hence for all $f \in H^{s}\left(\mu_{k}\right)$, one has $\left\|\mathscr{F}_{k}(f)\right\|_{L^{2}\left(\mu_{k}\right)} \leq$ $\|f\|_{H^{s}\left(\mu_{k}\right)}$, and by Hölder's inequality

$$
\left\|\mathscr{F}_{k}(f)\right\|_{L^{1}\left(\mu_{k}\right)} \leq\left[\int_{\mathbb{R}^{d}} \frac{d \mu_{k}(z)}{\left(1+|z|^{2}\right)^{s}}\right]^{1 / 2}\|f\|_{H^{s}\left(\mu_{k}\right)} .
$$

Then the function $\mathscr{F}_{k}(f)$ belongs to $L^{1} \cap L^{2}\left(\mu_{k}\right)$, and therefore

$$
\begin{array}{r}
f(x)=\int_{\mathbb{R}^{d}} E_{k}(i x, z) \mathscr{F}_{k}(f)(z) d \mu_{k}(z), \\
\text { a.e. } x \in \mathbb{R}^{d} .
\end{array}
$$

Let $\lambda>0$. We denote by $\langle\cdot, \cdot\rangle_{\lambda, H^{s}\left(\mu_{k}\right)}$ the inner product defined on the space $H^{s}\left(\mu_{k}\right)$ by

$$
\langle f, g\rangle_{\lambda, H^{s}\left(\mu_{k}\right)}:=\lambda\langle f, g\rangle_{H^{s}\left(\mu_{k}\right)}+\left\langle T_{k, m} f, T_{k, m} g\right\rangle_{L^{2}\left(\Omega_{k}\right)},
$$

and the norm $\|f\|_{\lambda, H^{s}\left(\mu_{k}\right)}:=\sqrt{\langle f, f\rangle_{\lambda, H^{s}\left(\mu_{k}\right)}}$.
Next we suppose that $m \in L^{2}\left(\mu_{k}\right)$ satisfying (22). By Theorem 3, the inner product $\langle\cdot, \cdot\rangle_{\lambda, H^{s}\left(\mu_{k}\right)}$ can be written as

$$
\langle f, g\rangle_{\lambda, H^{s}\left(\mu_{k}\right)}=\lambda\langle f, g\rangle_{H^{s}\left(\mu_{k}\right)}+\langle f, g\rangle_{L^{2}\left(\mu_{k}\right)} .
$$

Theorem 10. Let $\lambda>0$ and $s>\gamma+d / 2$ and let $m \in$ $L^{2}\left(\mu_{k}\right)$ satisfying $(22)$. The space $\left(H^{s}\left(\mu_{k}\right),\langle\cdot, \cdot\rangle_{\lambda, H^{s}\left(\mu_{k}\right)}\right)$ has the reproducing kernel

$$
K_{s}(x, y)=\int_{\mathbb{R}^{d}} \frac{E_{k}(i x, z) E_{k}(-i y, z)}{1+\lambda\left(1+|z|^{2}\right)^{s}} d \mu_{k}(z) ;
$$

that is,

(i) for all $y \in \mathbb{R}^{d}$, the function $x \rightarrow K_{s}(x, y)$ belongs to $H^{s}\left(\mu_{k}\right)$;

(ii) the reproducing property, for all $f \in H^{s}\left(\mu_{k}\right)$ and $y \in$ $\mathbb{R}^{d}$, is

$$
\left\langle f, K_{s}(\cdot, y)\right\rangle_{\lambda, H^{s}\left(\mu_{k}\right)}=f(y) .
$$

Proof. (i) Let $y \in \mathbb{R}^{d}$. From (12), the function $\Phi_{y}: z \rightarrow$ $E_{k}(-i y, z) /\left(1+\lambda\left(1+|z|^{2}\right)^{s}\right)$ belongs to $L^{1} \cap L^{2}\left(\mu_{k}\right)$. Then, the function $K_{s}$ is well defined and by Theorem 1(ii), we have

$$
K_{s}(x, y)=\mathscr{F}_{k}^{-1}\left(\Phi_{y}\right)(x), \quad x \in \mathbb{R}^{d} .
$$

From Theorem 1(iii), it follows that $K_{s}(\cdot, y)$ belongs to $L^{2}\left(\mu_{k}\right)$, and we have

$$
\mathscr{F}_{k}\left(K_{s}(\cdot, y)\right)(z)=\frac{E_{k}(-i y, z)}{1+\lambda\left(1+|z|^{2}\right)^{s}}, \quad z \in \mathbb{R}^{d} .
$$

Then by (12), we obtain

$$
\begin{aligned}
\left|\mathscr{F}_{k}\left(K_{s}(\cdot, y)\right)(z)\right| & \leq \frac{1}{\lambda\left(1+|z|^{2}\right)^{s}}, \\
\left\|K_{s}(\cdot, y)\right\|_{H^{s}\left(\mu_{k}\right)}^{2} & \leq \frac{1}{\lambda^{2}} \int_{\mathbb{R}^{d}} \frac{d \mu_{k}(z)}{\left(1+|z|^{2}\right)^{s}}<\infty .
\end{aligned}
$$

This proves that for all $y \in \mathbb{R}^{d}$ the function $K_{s}(\cdot, y)$ belongs to $H^{s}\left(\mu_{k}\right)$.

(ii) Let $f \in H^{s}\left(\mu_{k}\right)$ and $y \in \mathbb{R}^{d}$. From (62) and (66), we have

$$
\begin{aligned}
&\left\langle f, K_{s}(\cdot, y)\right\rangle_{\lambda, H^{s}\left(\mu_{k}\right)} \\
& \quad=\int_{\mathbb{R}^{d}} E_{k}(i y, z) \mathscr{F}_{k}(f)(z) d \mu_{k}(z),
\end{aligned}
$$

and from Remark 9, we obtain the following reproducing property:

$$
\left\langle f, K_{s}(\cdot, y)\right\rangle_{\lambda, H^{s}\left(\mu_{k}\right)}=f(y) .
$$

This completes the proof of the theorem. 
4.2. Tikhonov Regularization. The main result of this subsection can then be stated as follows.

Theorem 11. Let $s>\gamma+d / 2$ and let $m \in L^{2}\left(\mu_{k}\right)$ satisfying (22). For any $g \in L^{2}\left(\Omega_{k}\right)$ and for any $\lambda>0$, there exists $a$ unique function $f_{\lambda, g}^{*}$, where the infimum

$$
\inf _{f \in H^{s}\left(\mu_{k}\right)}\left\{\lambda\|f\|_{H^{s}\left(\mu_{k}\right)}^{2}+\left\|g-T_{k, m} f\right\|_{L^{2}\left(\Omega_{k}\right)}^{2}\right\}
$$

is attained. Moreover, the extremal function $f_{\lambda, g}^{*}$ is given by

$$
\begin{gathered}
f_{\lambda, g}^{*}(y) \\
=\int_{\mathbb{R}^{d}} \int_{0}^{\infty} \int_{\mathbb{R}^{d}} g(a, x) \frac{\overline{m(a z)} E_{k}(-i x, z) E_{k}(i y, z)}{1+\lambda\left(1+|z|^{2}\right)^{s}} \\
\cdot d \mu_{k}(z) d \Omega_{k}(a, x) .
\end{gathered}
$$

Proof. The existence and unicity of the extremal function $f_{\lambda, g}^{*}$ satisfying (70) are given by Kimeldorf and Wahba [19], Matsuura et al. [11], and Saitoh [20]. Moreover, by Theorem 10 we deduce that

$$
f_{\lambda, g}^{*}(y)=\left\langle g, T_{k, m}\left(K_{s}(\cdot, y)\right)\right\rangle_{L^{2}\left(\Omega_{k}\right)},
$$

where $K_{s}$ is the kernel given by (63).

But by Theorems 1(ii) and (66), we have

$$
\begin{aligned}
T_{k, m} & \left(K_{s}(\cdot, y)\right)(a, x) \\
= & \int_{\mathbb{R}^{d}} m(a z) \mathscr{F}_{k}\left(K_{s}(\cdot, y)\right)(z) E_{k}(i x, z) d \mu_{k}(z) \\
= & \int_{\mathbb{R}^{d}} m(a z) \frac{E_{k}(i x, z) E_{k}(-i y, z)}{1+\lambda\left(1+|z|^{2}\right)^{s}} d \mu_{k}(z) .
\end{aligned}
$$

This clearly yields the result.

Remark 12. The extremal function $f_{\lambda, g}^{*}$ satisfies the following inequality:

$$
\left|f_{\lambda, g}^{*}(y)\right| \leq \frac{\|g\|_{L^{2}\left(\Omega_{k}\right)}}{2 \sqrt{\lambda}}\left[\int_{\mathbb{R}^{d}} \frac{d \mu_{k}(z)}{\left(1+|z|^{2}\right)^{s}}\right]^{1 / 2} .
$$

Proof. From (72) and Theorem 3, we have

$$
\begin{aligned}
\left|f_{\lambda, g}^{*}(y)\right| & \leq\|g\|_{L^{2}\left(\Omega_{k}\right)}\left\|T_{k, m}\left(K_{s}(\cdot, y)\right)\right\|_{L^{2}\left(\Omega_{k}\right)} \\
& \leq\|g\|_{L^{2}\left(\Omega_{k}\right)}\left\|K_{s}(\cdot, y)\right\|_{L^{2}\left(\mu_{k}\right)} .
\end{aligned}
$$

Then, by Theorems 1(iii) and (66),

$$
\begin{aligned}
& \left|f_{\lambda, g}^{*}(y)\right| \\
& \quad \leq\|g\|_{L^{2}\left(\Omega_{k}\right)}\left\|\mathscr{F}_{k}\left(K_{s}(\cdot, y)\right)\right\|_{L^{2}\left(\mu_{k}\right)} \\
& \quad \leq\|g\|_{L^{2}\left(\Omega_{k}\right)}\left[\int_{\mathbb{R}^{d}} \frac{d \mu_{k}(z)}{\left[1+\lambda\left(1+|z|^{2}\right)^{s}\right]^{2}}\right]^{1 / 2} .
\end{aligned}
$$

Using the fact that $\left[1+\lambda\left(1+|z|^{2}\right)^{s}\right]^{2} \geq 4 \lambda\left(1+|z|^{2}\right)^{s}$, we obtain the result.

If we take in (72), $g=T_{k, m} f$, where $f \in H^{s}\left(\mu_{k}\right)$, we denote by $f_{\lambda}^{*}=f_{\lambda, T_{k, m} f}^{*}$.

Then by Theorem 3, we have

$$
\begin{aligned}
f_{\lambda}^{*}(y) & =\left\langle T_{k, m} f, T_{k, m}\left(K_{s}(\cdot, y)\right)\right\rangle_{L^{2}\left(\Omega_{k}\right)} \\
& =\left\langle f, K_{s}(\cdot, y)\right\rangle_{L^{2}\left(\mu_{k}\right)} .
\end{aligned}
$$

Corollary 13. Let $s>\gamma+d / 2, f \in H^{s}\left(\mu_{k}\right)$, and $\lambda>0$. The extremal function $f_{\lambda}^{*}$ given by (77) satisfies the following properties:

(i) $f_{\lambda}^{*}(y)=\int_{\mathbb{R}^{d}} E_{k}(i y, z)\left(\mathscr{F}_{k}(f)(z) /(1+\lambda(1+\right.$ $\left.\left.\left.|z|^{2}\right)^{s}\right)\right) d \mu_{k}(z)$

(ii) $\mathscr{F}_{k}\left(f_{\lambda}^{*}\right)(z)=\mathscr{F}_{k}(f)(z) /\left(1+\lambda\left(1+|z|^{2}\right)^{s}\right)$;

(ii) $\left\|f_{\lambda}^{*}\right\|_{H^{s}\left(\mu_{k}\right)} \leq(1 / 2 \sqrt{\lambda})\|f\|_{L^{2}\left(\mu_{k}\right)}$.

Proof. (i) It follows from (77) by using Theorems 1(iii) and (66).

(ii) The function $F: z \rightarrow \mathscr{F}_{k}(f)(z) /\left(1+\lambda\left(1+|z|^{2}\right)^{s}\right)$ belongs to $L^{1} \cap L^{2}\left(\mu_{k}\right)$. Then by Theorem 1(ii), we have

$$
f_{\lambda}^{*}(y)=\mathscr{F}_{k}^{-1}(F)(y) .
$$

From Theorem 1(iii), it follows that $f_{\lambda}^{*}$ belongs to $L^{2}\left(\mu_{k}\right)$, and

$$
\mathscr{F}_{k}\left(f_{\lambda}^{*}\right)(z)=\frac{\mathscr{F}_{k}(f)(z)}{1+\lambda\left(1+|z|^{2}\right)^{s}} .
$$

(iii) By relation (ii) we have

$$
\begin{aligned}
& \left\|f_{\lambda}^{*}\right\|_{H^{s}\left(\mu_{k}\right)}^{2} \\
& =\int_{\mathbb{R}^{d}}\left(1+|z|^{2}\right)^{s}\left|\mathscr{F}_{k}\left(f_{\lambda}^{*}\right)(z)\right|^{2} d \mu_{k}(z) \\
& =\int_{\mathbb{R}^{d}} \frac{\left(1+|z|^{2}\right)^{s}}{\left[1+\lambda\left(1+|z|^{2}\right)^{s}\right]^{2}}\left|\mathscr{F}_{k}(f)(z)\right|^{2} d \mu_{k}(z) .
\end{aligned}
$$

Using inequality $\left[1+\lambda\left(1+|z|^{2}\right)^{s}\right]^{2} \geq 4 \lambda\left(1+|z|^{2}\right)^{s}$, we obtain

$$
\left\|f_{\lambda}^{*}\right\|_{H^{s}\left(\mu_{k}\right)}^{2} \leq \frac{1}{4 \lambda} \int_{\mathbb{R}^{d}}\left|\mathscr{F}_{k}(f)(z)\right|^{2} d \mu_{k}(z)=\frac{1}{4 \lambda}\|f\|_{L^{2}\left(\mu_{k}\right)}^{2} .
$$

This completes the proof of the corollary.

Corollary 14. Let $s>\gamma+d / 2, f \in H^{s}\left(\mu_{k}\right)$, and $\lambda>0$. The extremal function $f_{\lambda}^{*}$ given by (77) satisfies

$$
\lim _{\lambda \rightarrow 0^{+}}\left\|f_{\lambda}^{*}-f\right\|_{H^{s}\left(\mu_{k}\right)}=0 \text {. }
$$

Moreover, $\left\{f_{\lambda}^{*}\right\}_{\lambda>0}$ converges uniformly to $f$ as $\lambda \rightarrow 0^{+}$. 
Proof. From Corollary 13(ii),

$$
\mathscr{F}_{k}\left(f_{\lambda}^{*}-f\right)(z)=\frac{-\lambda\left(1+|z|^{2}\right)^{s}}{1+\lambda\left(1+|z|^{2}\right)^{s}} \mathscr{F}_{k}(f)(z) .
$$

Consequently,

$$
\begin{aligned}
& \left\|f_{\lambda}^{*}-f\right\|_{H^{s}\left(\mu_{k}\right)}^{2} \\
& \quad=\int_{\mathbb{R}^{d}} \frac{\lambda^{2}\left(1+|z|^{2}\right)^{3 s}\left|\mathscr{F}_{k}(f)(z)\right|^{2}}{\left[1+\lambda\left(1+|z|^{2}\right)^{s}\right]^{2}} d \mu_{k}(z) .
\end{aligned}
$$

Using the dominated convergence theorem and the fact that

$$
\begin{gathered}
\frac{\lambda^{2}\left(1+|z|^{2}\right)^{3 s}\left|\mathscr{F}_{k}(f)(z)\right|^{2}}{\left[1+\lambda\left(1+|z|^{2}\right)^{s}\right]^{2}} \\
\leq\left(1+|z|^{2}\right)^{s}\left|\mathscr{F}_{k}(f)(z)\right|^{2},
\end{gathered}
$$

we deduce that

$$
\lim _{\lambda \rightarrow 0^{+}}\left\|f_{\lambda}^{*}-f\right\|_{H^{s}\left(\mu_{k}\right)}^{2}=0 .
$$

On the other hand, from Remark 9, the function $\mathscr{F}_{k}(f) \in$ $L^{1} \cap L^{2}\left(\mu_{k}\right)$. Then by (83) and Theorem 1(ii),

$$
\begin{aligned}
& f_{\lambda}^{*}(y)-f(y) \\
& \quad=\int_{\mathbb{R}^{d}} \frac{-\lambda\left(1+|z|^{2}\right)^{s} \mathscr{F}_{k}(f)(z)}{1+\lambda\left(1+|z|^{2}\right)^{s}} E_{k}(i y, z) d \mu_{k}(z) .
\end{aligned}
$$

So

$$
\begin{aligned}
& \left\|f_{\lambda}^{*}-f\right\|_{L^{\infty}\left(\mu_{k}\right)} \\
& \quad \leq \int_{\mathbb{R}^{d}} \frac{\lambda\left(1+|z|^{2}\right)^{s}\left|\mathscr{F}_{k}(f)(z)\right|}{1+\lambda\left(1+|z|^{2}\right)^{s}} d \mu_{k}(z) .
\end{aligned}
$$

Again, by dominated convergence theorem and the fact that

$$
\frac{\lambda\left(1+|z|^{2}\right)^{s}\left|\mathscr{F}_{k}(f)(z)\right|}{1+\lambda\left(1+|z|^{2}\right)^{s}} \leq\left|\mathscr{F}_{k}(f)(z)\right|,
$$

we deduce that

$$
\lim _{\lambda \rightarrow 0^{+}}\left\|f_{\lambda}^{*}-f\right\|_{L^{\infty}\left(\mu_{k}\right)}=0,
$$

which ends the proof.

As application, we give the following examples.

Example 15. If $m_{t}(x):=-\sqrt{8} t|x|^{2} e^{-t|x|^{2}}$ and $s>\gamma+d / 2$, then

$$
\begin{gathered}
f_{\lambda, g}^{*}(y) \\
=-\sqrt{8} t \int_{\mathbb{R}^{d}} \int_{0}^{\infty} \int_{\mathbb{R}^{d}} \frac{a^{2} e^{-t a^{2}|z|^{2}}|z|^{2} g(a, x)}{1+\lambda\left(1+|z|^{2}\right)^{s}} \\
\cdot E_{k}(-i x, z) E_{k}(i y, z) d \mu_{k} \\
\cdot(z) d \Omega_{k}(a, x),
\end{gathered}
$$

and by (28), (72), and the fact that $K_{s}(y, z)=\overline{K_{s}(z, y)}$ we obtain

$$
\begin{aligned}
& f_{\lambda, g}^{*}(y) \\
& =\sqrt{8} t \int_{\mathbb{R}^{d}} \int_{0}^{\infty} \int_{\mathbb{R}^{d}} \frac{g(a, x)}{a^{2 \gamma+d}} \frac{\partial}{\partial t}\left[h_{k}\left(\frac{x}{a}, \frac{z}{a}, t\right)\right] \\
& \cdot K_{s}(y, z) d \mu_{k}(z) d \Omega_{k}(a, x) .
\end{aligned}
$$

Example 16. If $m_{t}(x):=-2 t|x| e^{-t|x|}$ and $s>\gamma+d / 2$, then

$$
\begin{gathered}
f_{\lambda, g}^{*}(y) \\
=-2 t \int_{\mathbb{R}^{d}} \int_{0}^{\infty} \int_{\mathbb{R}^{d}} \frac{a e^{-t a|z|}|z| g(a, x)}{1+\lambda\left(1+|z|^{2}\right)^{s}} \\
\cdot E_{k}(-i x, z) E_{k}(i y, z) d \mu_{k} \\
\cdot(z) d \Omega_{k}(a, x),
\end{gathered}
$$

and by (39) and (72) we deduce that

$$
\begin{aligned}
& f_{\lambda, g}^{*}(y) \\
& =2 t \int_{\mathbb{R}^{d}} \int_{0}^{\infty} \int_{\mathbb{R}^{d}} \frac{g(a, x)}{a^{2 \gamma+d}} \frac{\partial}{\partial t}\left[p_{k}\left(\frac{x}{a}, \frac{z}{a}, t\right)\right] \\
& \cdot K_{s}(y, z) d \mu_{k}(z) d \Omega_{k}(a, x) .
\end{aligned}
$$

\section{Conflict of Interests}

The author declares that there is no conflict of interests regarding the publication of this paper.

\section{Acknowledgment}

The author is partially supported by Deanship of Scientific Research, Jazan University.

\section{References}

[1] C. F. Dunkl, "Hankel transforms associated to finite reflection groups," Contemporary Mathematics, vol. 138, pp. 123-138, 1992.

[2] M. Rösler, "An uncertainty principle for the Dunkl transform," Bulletin of the Australian Mathematical Society, vol. 59, no. 3, pp. 353-360, 1999.

[3] N. Shimeno, "A note on the uncertainty principle for the Dunkl transform," The University of Tokyo. Journal of Mathematical Sciences, vol. 8, no. 1, pp. 33-42, 2001.

[4] F. Soltani, "Heisenberg-Pauli-Weyl uncertainty ine quality for the Dunkl transform on $\mathbb{R}^{d}$," Bulletin of the Australian Mathematical Society, vol. 87, no. 2, pp. 316-325, 2013.

[5] F. Soltani, "A general form of Heisenberg-Pauli-Weyl uncertainty inequality for the Dunkl transform," Integral Transforms and Special Functions, vol. 24, no. 5, pp. 401-409, 2013.

[6] F. Soltani, "Best approximation formulas for the Dunkl L2multiplier operators on $\mathrm{R}^{d}$," The Rocky Mountain Journal of Mathematics, vol. 42, no. 1, pp. 305-328, 2012. 
[7] F. Soltani, "Multiplier operators and extremal functions related to the dual Dunkl-Sonine operator," Acta Mathematica Scientia. Series B: English Edition, vol. 33, no. 2, pp. 430-442, 2013.

[8] D. L. Donoho and P. B. Stark, "Uncertainty principles and signal recovery," SIAM Journal on Applied Mathematics, vol. 49, no. 3, pp. 906-931, 1989.

[9] S. Saitoh, "The Weierstrass transform and an isometry in the heat equation," Applicable Analysis, vol. 16, no. 1, pp. 1-6, 1983.

[10] S. Saitoh, "Best approximation, Tikhonov regularization and reproducing kernels," Kodai Mathematical Journal, vol. 28, no. 2, pp. 359-367, 2005.

[11] T. Matsuura, S. Saitoh, and D. D. Trong, "Approximate and analytical inversion formulas in heat conduction on multidimensional spaces," Journal of Inverse and Ill-Posed Problems, vol. 13, no. 3-6, pp. 479-493, 2005.

[12] M. Yamada, T. Matsuura, and S. Saitoh, "Representations of inverse functions by the integral transform with the sign kernel," Fractional Calculus \& Applied Analysis, vol. 10, no. 2, pp. 161-168, 2007.

[13] C. F. Dunkl, "Integral kernels with reflection group invariance," Canadian Journal of Mathematics, vol. 43, no. 6, pp. 1213-1227, 1991.

[14] M. F. E. de Jeu, “The Dunkl transform,” Inventiones Mathematicae, vol. 113, no. 1, pp. 147-162, 1993.

[15] E. M. Opdam, "Dunkl operators, Bessel functions and the discriminant of a finite Coxeter group," Compositio Mathematica, vol. 85, no. 3, pp. 333-373, 1993.

[16] M. Rösler and M. Voit, "Markov processes related with Dunkl operators," Advances in Applied Mathematics, vol. 21, no. 4, pp. 575-643, 1998.

[17] F. Soltani, "Inversion formulas in the Dunkl -type heat conduction on R d," Applicable Analysis, vol. 84, no. 6, pp. 541-553, 2005.

[18] F. Soltani, "Littlewood-Paley $g$-function in the Dunkl analysis on $\mathrm{R}^{d}$," Journal of Inequalities in Pure and Applied Mathematics, vol. 6, no. 3, article 84, 13 pages, 2005.

[19] G. Kimeldorf and G. Wahba, "Some results on Tchebycheffian spline functions," Journal of Mathematical Analysis and Applications, vol. 33, pp. 82-95, 1971.

[20] S. Saitoh, "Approximate real inversion formulas of the Gaussian convolution," Applicable Analysis, vol. 83, no. 7, pp. 727-733, 2004. 


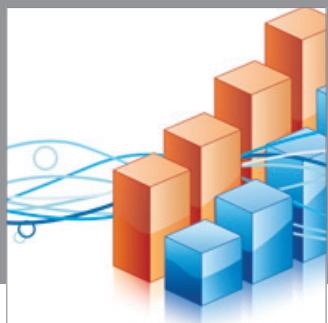

Advances in

Operations Research

mansans

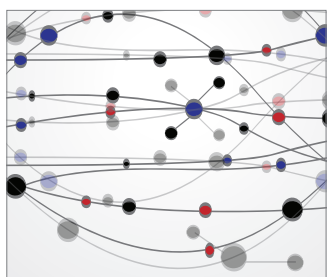

The Scientific World Journal
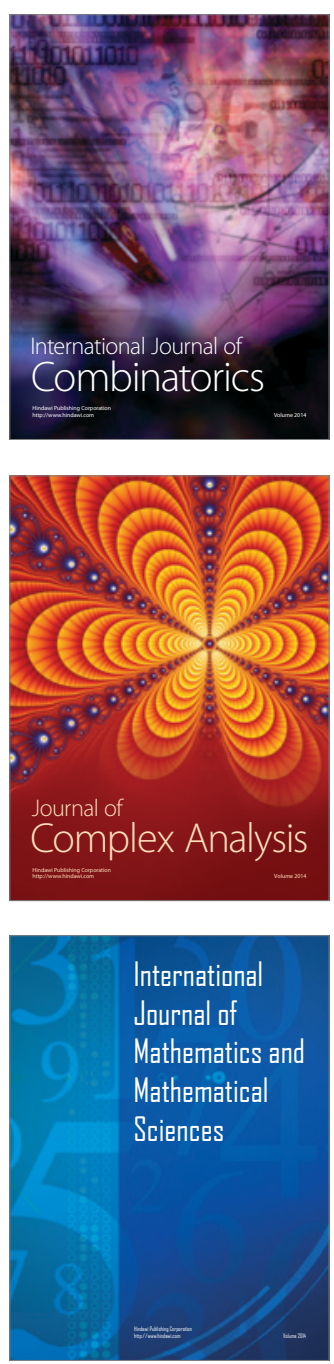
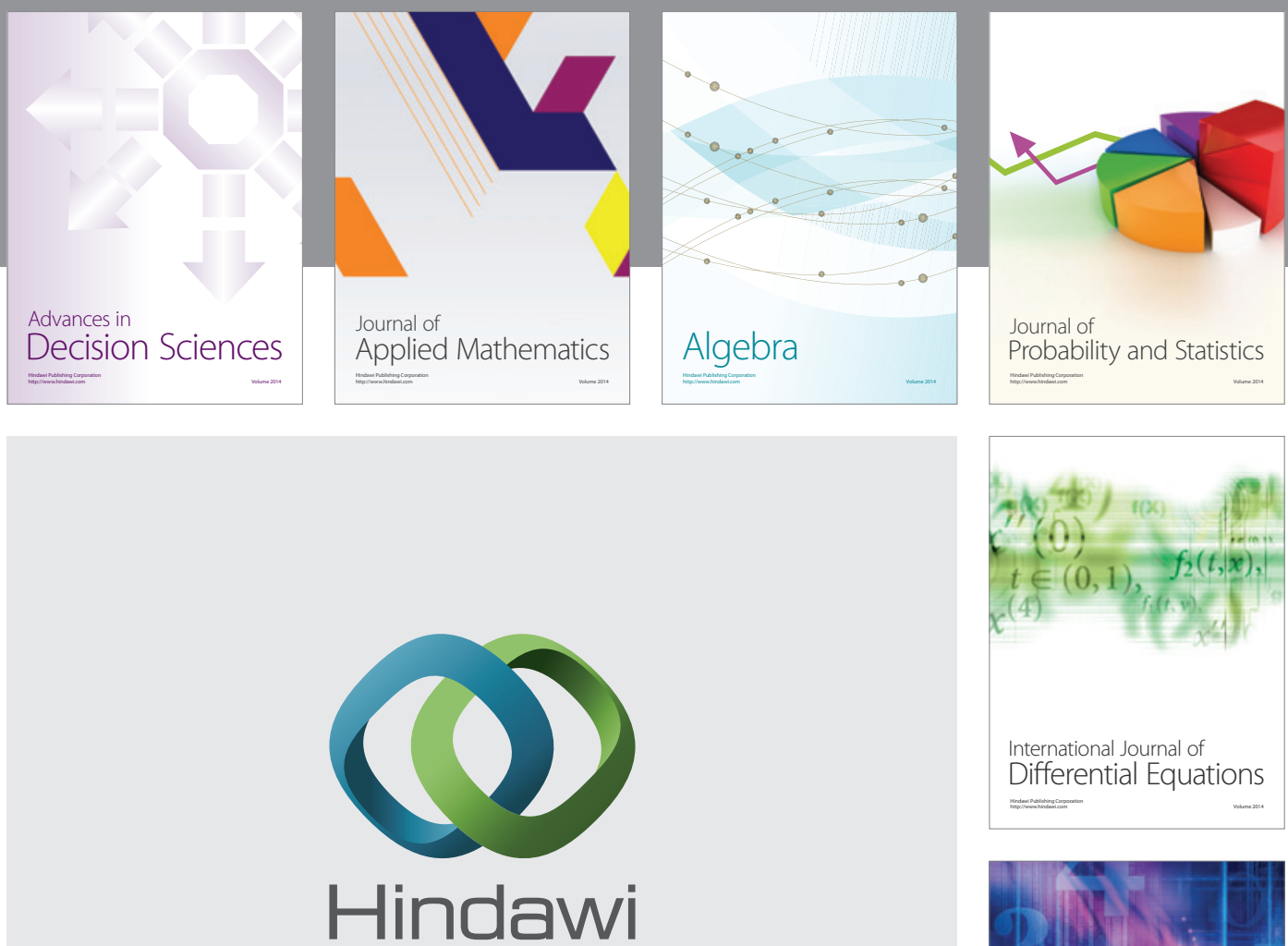

Submit your manuscripts at http://www.hindawi.com
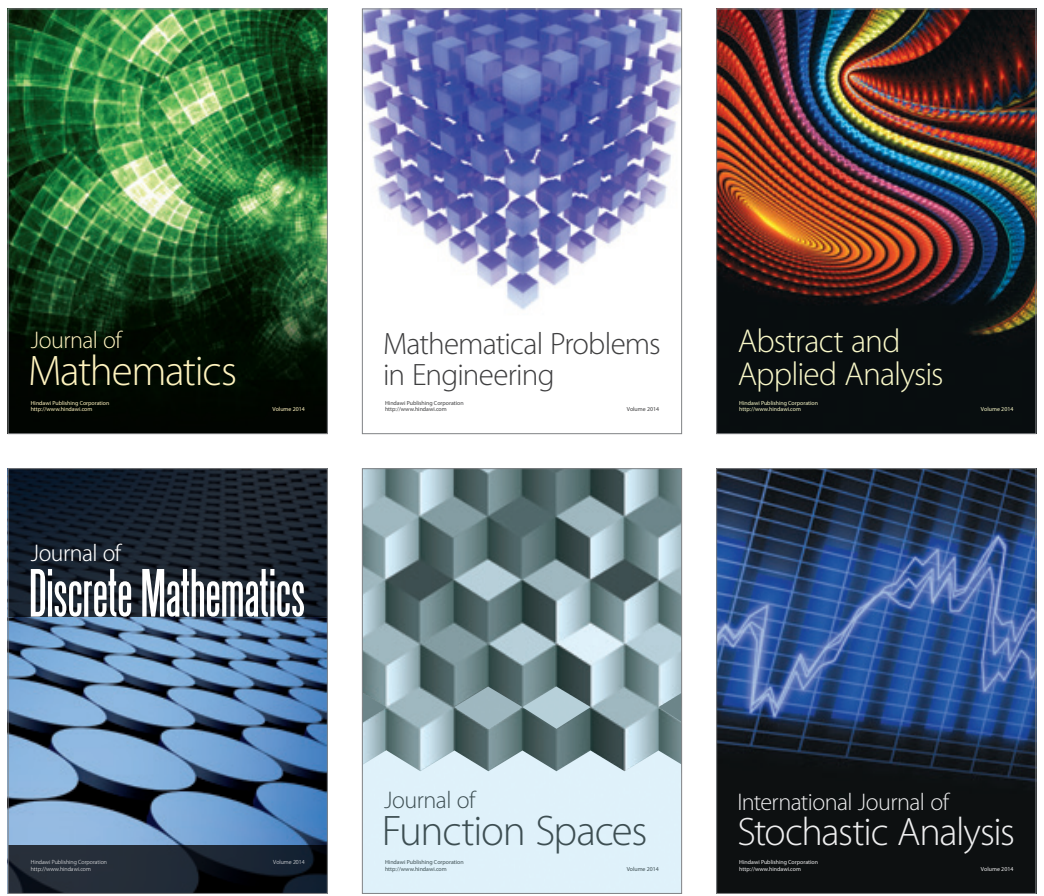

Journal of

Function Spaces

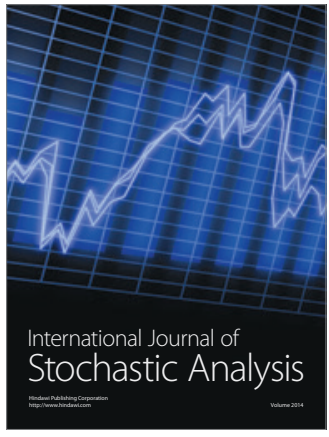

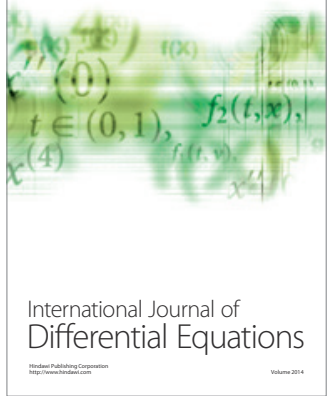
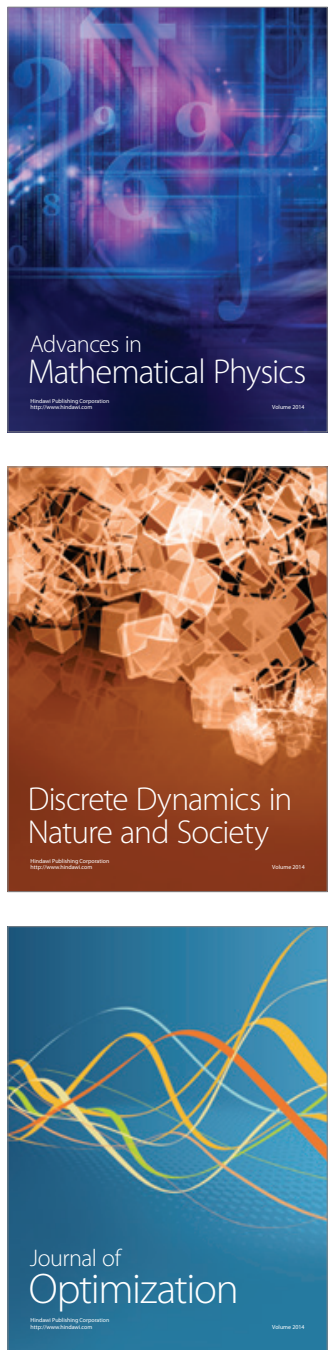